\title{
The Use of Induced Pluripotent Stem Cell Technology to Advance Autism Research and Treatment
}

\author{
Allan Acab ${ }^{1}$ - Alysson Renato Muotri ${ }^{1}$ \\ Published online: 8 April 2015 \\ (C) The American Society for Experimental NeuroTherapeutics, Inc. 2015
}

\begin{abstract}
Autism spectrum disorders (ASDs) are a heterogeneous group of neurodevelopmental disorders sharing a core set of symptoms, including impaired social interaction, language deficits, and repetitive behaviors. While ASDs are highly heritable and demonstrate a clear genetic component, the cellular and molecular mechanisms driving ASD etiology remain undefined. The unavailability of live patient-specific neurons has contributed to the difficulty in studying ASD pathophysiology. The recent advent of induced pluripotent stem cells (iPSCs) has provided the ability to generate patient-specific human neurons from somatic cells. The iPSC field has quickly grown, as researchers have demonstrated the utility of this technology to model several diseases, especially neurologic disorders. Here, we review the current literature around using iPSCs to model ASDs, and discuss the notable findings, and the promise and limitations of this technology. The recent report of a nonsyndromic ASD iPSC model and several previous ASD models demonstrating similar results points to the ability of iPSC to reveal potential novel biomarkers and therapeutics.
\end{abstract}

Keywords Disease modeling $\cdot$ Human induced pluripotent stem cells $\cdot$ Human neurons $\cdot$ Brain $\cdot$ Drug screening $\cdot$ Autism spectrum disorders

Electronic supplementary material The online version of this article (doi:10.1007/s13311-015-0354-x) contains supplementary material, which is available to authorized users.

Alysson Renato Muotri

muotri@ucsd.edu

1 School of Medicine, Department of Pediatrics/Rady Children's Hospital San Diego, Department of Cellular and Molecular Medicine, Stem Cell Program, University of California San Diego, MC 0695, La Jolla, CA 92093, USA

\section{Introduction}

Autism spectrum disorders (ASDs) are a set of complex neurodevelopmental disorders sharing a core set of symptoms, including social impairments, communication deficits, and stereotyped repetitive behaviors [1]. According to the new Diagnostic and Statistical Manual of Mental Disorders, 5th Edition, classifications, once diagnosed separately, cases of autism, Asperger syndrome, and pervasive developmental disorders are now classified under the umbrella term "autism spectrum disorders" [2]. While the exact etiology of these ASDs remains unknown, ASDs do demonstrate a strong genetic component $[3,4]$. Previously, ASDs had also been categorized as syndromic (caused by a known genetic disorder) or nonsyndromic (idiopathic, unknown genetic cause). Although not specifically identified in the Diagnostic and Statistical Manual of Mental Disorders, 5th Edition, nomenclature, such distinctions help to further classify and understand different ASD cases based on genetic etiology. These neurodevelopmental syndromes that also manifest autistic symptoms (which we will refer to here as syndromic ASDs) include Rett syndrome (RTT), Timothy syndrome (TS), fragile X syndrome (FXS), Angelman syndrome (AS), and Phelan-McDermid syndrome (PMDS). These syndromic ASDs are caused by defined genetic or chromosomal abnormalities, and are estimated to account for 10-20\% of ASD cases [5]. The genetic abnormalities associated with nonsyndromic ASDs, which make up the majority of ASD cases, are being intensively researched, with evidence for both hereditary and de novo mutations [6-9]. Several different chromosomal loci and genetic variants have been implicated in ASD susceptibility, indicating that while symptoms are shared, these disorders are genetically heterogeneous [4]. Increasing numbers of rare variants are being implicated in ASD, and often presenting modest-to-low degrees of risk [10]. These studies support the multiple-hit hypothesis of 
autism, which postulates that some nonsyndromic ASDs are caused by a combination of several genetic abnormalities affecting specific pathways above a threshold level [11-13]. However, while several ASD-related genes have been implicated, the functional study of these genes and their individual relevance to human ASD etiology remains lacking. Thus, the identification and analysis of the functional relevance and cellular contributions of these ASD variants is critical for the elucidation of autism pathophysiology.

The lack of relevant human disease models has hindered the understanding of ASD etiology. Until recently, human neurologic disorder researchers have lacked sufficient amounts of samples to properly study the target cell type, the neuron. Access to the affected cell type is essential for the analysis of cellular and molecular mechanisms driving the disorders. Human postmortem samples have long been used to study phenotypes of neurological disorders but often present several limitations [14]. These samples often represent only the end stage of the disease, where secondary symptoms and phenotypes can present problems [15]. Even more, environmental factors such as drug treatments can also play confounding roles. In addition, the obvious lack of living cells in preserved postmortem samples essentially prohibits the use of functional assays to study cellular physiology and neural networks.

Animal models have also been long used to model neurologic diseases and disorders to study disease etiology $[16,17]$. Transgenic and knockout technology using mouse models can provide valuable analysis of genetic disorders in vivo and in vitro. However, they are restricted mostly to monogenetic diseases, which is limiting for genetically complex, heterogeneous disorders such as autism. Disorders characterized by several rare variants, translocations, or large deletions are difficult to model in mice, especially when considering species differences in genetics. In addition, mouse models often do not fully recapitulate complex human diseases, especially social and behavioral disorders such as autism.

Thus, to understand cellular and molecular phenotypes driving neuropsychiatric disorders such as ASDs, a human neuronal cellular model able to both recapitulate the causal genetics and produce the target cell type is necessary. The advancement of stem cell technology has allowed for the generation of these human cellular models. Pluripotent human embryonic stem cells (ESCs) arose as promising sources of human cells, able to study early developmental time points, as well as generate multiple cell types. However, ethical issues and the scarcity of available disease-specific human ESCs lines have hindered disease modeling progress. The advent of cellular genetic reprogramming has revolutionized human cellular disease modeling. Recently developed, somatic cells such as fibroblasts and dental pulp cells can be reprogrammed into a pluripotent state by the overexpression of specific transcription factors [18]. These induced pluripotent stem cells
(iPSCs), can then be differentiated into virtually any target cell type. These iPSCs are isogenic to the original donor cells, and thus recapitulate the genetics of the patient from which they were obtained. Previously not possible, unlimited numbers of human cells such as neurons, even carrying disease-specific mutations, can be generated. Researchers are then able to examine cellular phenotypes, perform functional assays, and test drugs for any potential efficacy in ameliorating defects.

In this review, we discuss recent iPSC disease models of autism, examine the noteworthy findings, and explore the future implications and challenges in using these human cellular models for understanding autism etiology.

\section{iPSC Disease Modeling}

Since the inception of iPSC technology, several diseases have been successfully modeled [19-21]. Virtually any disorder known to have some genetic basis can be modeled by iPSCs; however, the successful identification of cellular phenotypes can be quite variable [22]. Furthermore, these human cellular models are particularly useful when no good animal model exists. Several human diseases affecting different human tissue types have been modeled, including hematopoietic disorders such as Fanconi anemia [23]. Cardiovascular disorders such as long QT syndrome and LEOPARD syndrome have also been successfully modeled [24-26]. Interestingly, while mouse models do not reproduce the human phenotypes, Itzhaki et al. [25] found long QT syndrome iPSC-derived cardiomyocytes to reproduce the prolonged action potential observed in patients. Importantly, the authors were able to perform a simple screen and demonstrate that $\beta$-blockers can improve the affected cardiomyocyte QT interval. These studies demonstrate the capability of iPSC models to recapitulate human phenotypes effectively and allow for the screening of drugs to ameliorate these defects.

While iPSCs can potentially generate any cell type, neurologic disorders have been the most frequent targets of iPSC disease modeling [27]. Neurodegenerative disorders such as amyotrophic lateral sclerosis (ALS), Parkinson disease (PD), and Alzheimer disease (AD) were among the first to be targeted using iPSCs [28-30]. While iPSC models of neurodegenerative disorders such as ALS and PD can generate neurons carrying disease-specific genetics, the lack of understanding of the mechanisms driving neurodegeneration undeniably makes phenotype identification using iPSCs more challenging. Unlike disorders such as long QT syndrome that have known hallmark defects, robust phenotypes for neurodegenerative disorders are scarce. Nevertheless, Dimos et al. [28] were able to generate iPSCs from patients with ALS, which could then differentiate into motor neurons and glia-cell types specifically affected in ALS. However, they were unable to observe any novel or robust cellular phenotypes. A 
subsequent study by Mitne-Neto et al. [31] modeled ALS8 using patients carrying a mutation of the vesicle-associated membrane protein (VAPB) protein. The authors revealed a potentially exploitable biochemical phenotype - that motor neurons generated from the VAPB-iPSC carried reduced protein levels of VAPB compared with controls. Soldner et al. [29] successfully modeled PD, a neurodegenerative disorder characterized by loss of dopaminergic neurons. The authors were able to generate dopaminergic neurons but failed to see any cellular phenotypes. However, subsequent iPSC models of PD were able to identify elevated oxidative stress in the iPSC-derived dopaminergic neurons [32]. AD results in progressive neuronal loss and while no clear disease etiology has been elucidated, hallmarks of the disease are the presence of $\beta$-amyloid $(\mathrm{A} \beta)$ plaques and neurofibrillary tangles [33]. The altered processing of $A \beta$ precursor protein into $A \beta$ peptides is thought to play a role in $\mathrm{AD}$ and the generation of plaques [34, 35]. Israel et al. [30] demonstrated that neurons generated from iPSCs from patients with AD produced elevated amounts of the pathogenic $A \beta$ peptide [30]. These reports suggest that iPSCs can provide an important model of neurodegenerative disorders to not only generate the affected human cell types, but also for the identification of mechanisms contributing to disease etiology.

Neurodevelopmental disorders have become a popular target of iPSC modeling, with recently published models of schizophrenia (SZ), Cockayne syndrome, and syndromic ASDs such as FXS, Down syndrome, and RTT [36-39]. Neurodevelopmental disorders are characterized by defects in central nervous system development and growth, and often have a genetic cause [40]. iPSC technology is particularly well suited to modeling genetic disorders because of its ability to capture disease-specific genotypes, which is especially useful for complex genetic disorders. As such, neurodevelopmental disorders are ideal targets because of their strong genetic component, with both monogenetic and complex multigenic forms [41]. In addition, unlike neurodegenerative conditions, neurodevelopmental disorders are often characterized by cellular defects apparent at early stages in life [40, 42].

For example, while SZ is distinct from early-onset neurodevelopmental disorders such as ASDs, in its later onset it can still be successfully modeled using iPSCs. SZ is a disabling neurologic disorder characterized by paranoia, hallucinations, and cognitive and emotional abnormalities [43]. SZ encompasses a spectrum of phenotypes, including neuroanatomic changes and altered neurotransmission across several neuronal subtypes [44]. While the spectrum of disease is broad and environmental conditions are important, evidence suggests SZ has a genetic basis [45-47]. The neurodevelopmental hypothesis of SZ suggests that the disease is caused by the altered interaction of multiple genes affecting important developmental pathways, inducing a cascade of neuropathologic changes and events during development [48-50]. Brennand et al. [36] generated iPSCs from 4 patients with SZ carrying complex genetic mutations [36]. Importantly, the authors were able to demonstrate that the neurons were less complex and contained fewer neurites, recapitulating postmortem studies [51, 52]. Furthermore, they showed other phenotypes, including reduced neuronal connectivity, synaptic protein levels, and altered gene expression. Moreover, neuronal connectivity and expression alterations were rescued after treatment with an antipsychotic drug, exemplifying the potential of iPSC models as drug discovery platforms.

\section{Modeling Syndromic Autism}

Syndromic forms of autism are the disorders falling under the umbrella of ASDs in which there is a known, usually monogenetic, cause. Unlike nonsyndromic, or idiopathic, forms of autism, where the genetic cause is unknown, syndromic forms are associated with specific genes that are known to cause an ASD when mutated. Because the genetic causes are already known, syndromic forms of ASD, including FXS, TS, cyclindependent kinase-like 5 disorder, and RTT, were quickly targeted for iPSC modeling (Table 1) [38, 39, 53, 54].

\section{FXS}

FXS is characterized by a CGG trinucleotide repeat expansion in the 5' untranslated region of FMR1 leading to hypermethylation and gene silencing [55]. FXS, which results when the expansion is $>200$ repeats, is the most common syndromic form of ASD in the population, and patients display with physical, intellectual, and behavioral phenotypes of varying severity [56, 57]. FMR1 encodes for the protein fragile $\mathrm{X}$ mental retardation protein (FMRP), which acts as an mRNA binding protein that regulates the translation of many genes, including those translated locally at the postsynaptic site. FMRP inhibits the translation of several mRNAs, and it was shown that loss of FMRP expression leads to increased numbers of spines and neuronal overexcitability [58]. An initial human ESC study of FXS demonstrated that FMR1 was unmethylated at the undifferentiated pluripotent stage, allowing for its expression [59]. However, the first reported iPSC FXS model showed that FMR1 remained inactive and retained the epigenetic silencing, highlighting differences between ESCs and iPSCs [38]. A subsequent study showed that multiple reprogrammed patient FXS lines had variable levels of FMR1 silencing and expression [60]. Highlighting its importance to neurodevelopment, lines that demonstrated reduced FRM1 expression resulted in aberrant neuronal differentiation [60]. Another study generated iPSC from FXS premutation individuals (carrying 55-200 CGG repeats), who do not display with classical FXS but suffer from neurodegenerative fragile $\mathrm{X}$-associated tremor/ataxia syndrome 


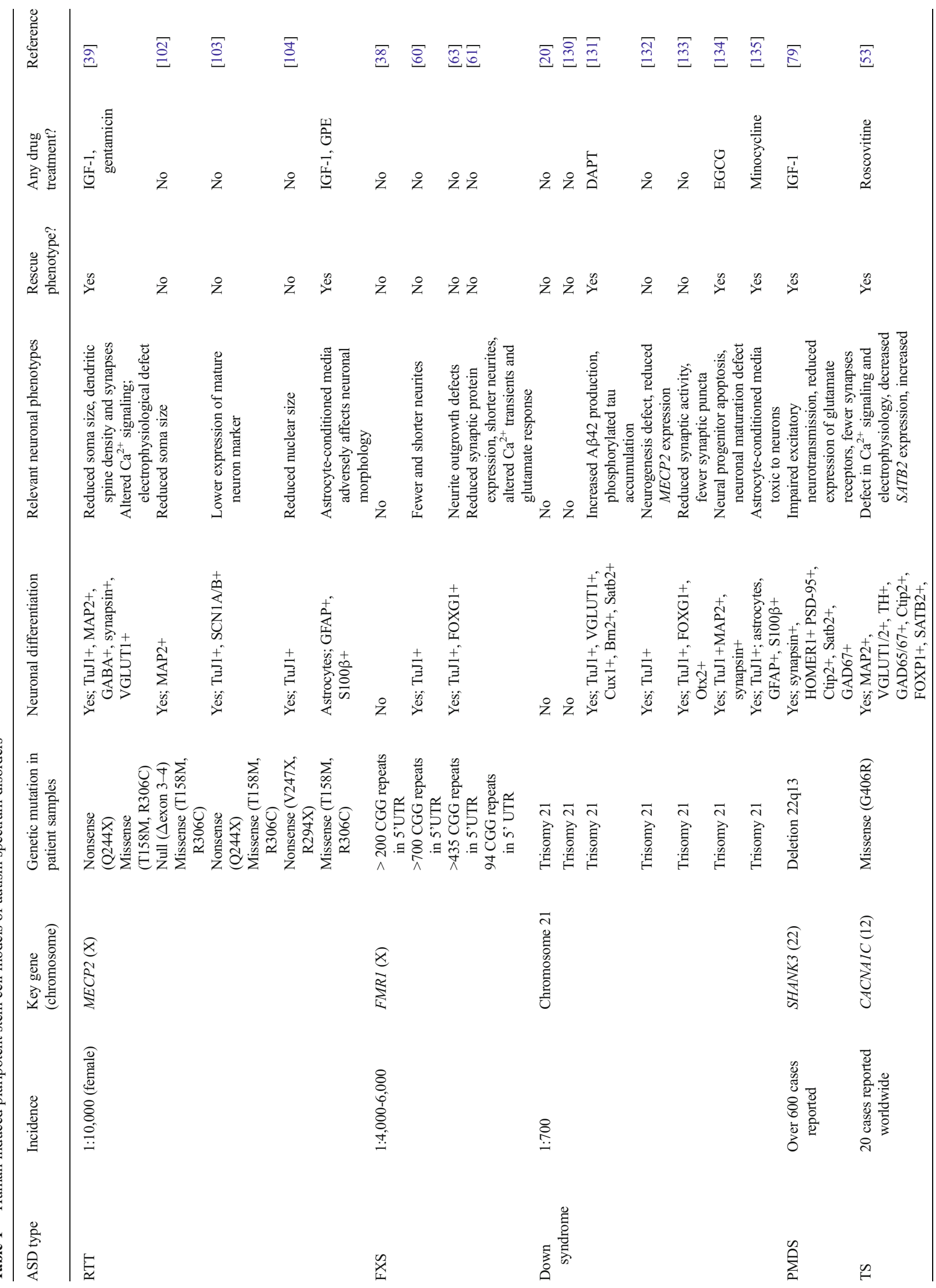




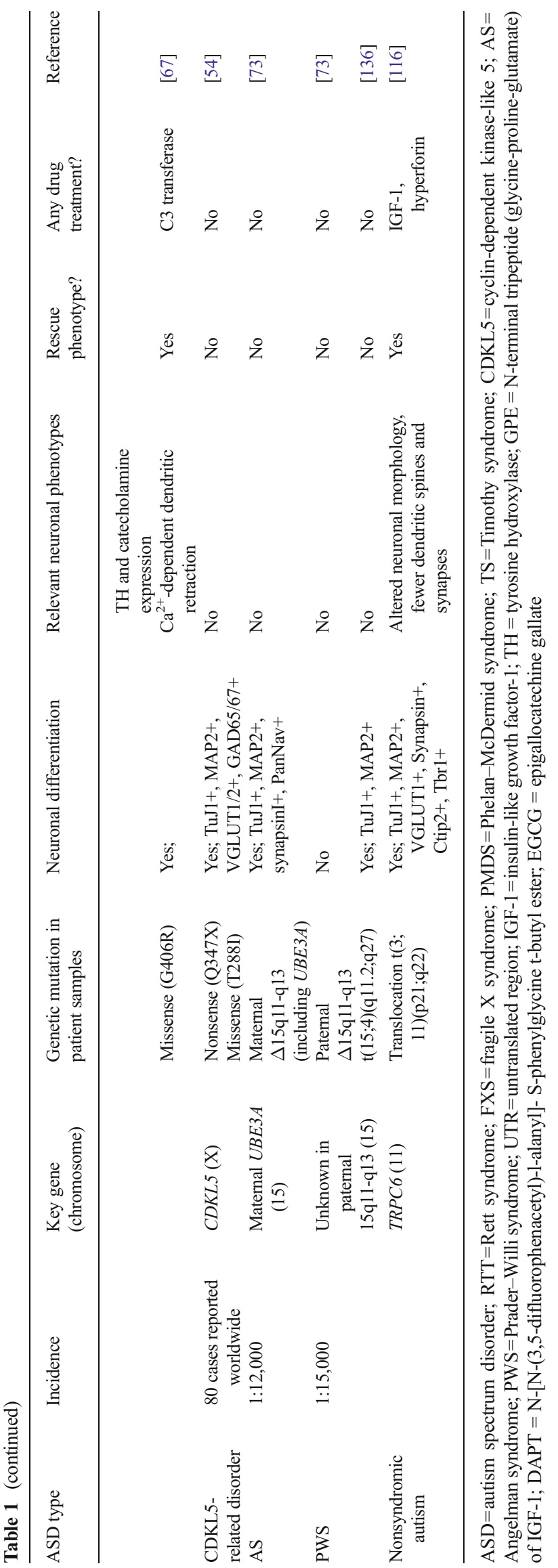

$[61,62]$. Interestingly, neurons derived from these iPSCs revealed reduced neurite length, fewer synaptic puncta, reduced synaptic protein levels, and increased calcium stransients [61]. A recent report showed that forebrain neurons derived from iPSCs from patients with FXS also showed reduced neurite outgrowth [63]. While facing initial hurdles, these reports represent the potential of FXS iPSC models to provide cellular tools that recapitulate disease phenotypes.

\section{TS}

TS is a rare autosomal dominant neurodevelopmental disorder caused by a mutation in $C A C N A 1 C$, which encodes for the voltage-dependent calcium channel $\mathrm{Ca}_{\mathrm{V}} 1.2$ [64]. TS has been associated with an array of phenotypic manifestations, including heart malformations, arrhythmia, developmental delay, and autism. The TS-causing mutation induces aberrant $\mathrm{Ca}_{\mathrm{V}} 1.2$ function, leading to loss of the voltage-dependent channel inactivation and subsequent excess of intracellular $\mathrm{Ca}^{2+}$. The high prevalence of patients with TS with ASD and intellectual disability underscores the importance of $\mathrm{Ca}_{\mathrm{V}} 1.2$ to neurodevelopment $[64,65]$. Pasca et al. [53] generated an iPSC model of TS, and identified several abnormalities in the derived cells, including neurons. Pasca et al. [53] reported defects in $\mathrm{Ca}^{2+}$ signaling, as well as defects in differentiation, with TS cells producing fewer neurons expressing cortical and callosal projection markers, and more neurons expressing tyrosine hydroxylase. While the connection between TS and ASD symptoms is still unclear, the authors claim the observed reduction in cortical projecting neurons is consistent with the connectivity hypothesis of ASD [66]. In addition, the increase in tyrosine hydroxylase-expressing neurons was ameliorated after treatment with the voltagedependent inactivation inhibitor roscovitine, highlighting the potential for a TS iPSC drug screening assay. A subsequent study of TS iPSCs revealed aberrant activity-dependent dendritic retraction in both the TS-derived neurons and rodent neurons [67]. The authors found that this was a result of RhoA activation and was independent of $\mathrm{Ca}^{2+}$ influx through $\mathrm{Ca}_{\mathrm{V}}$ 1.2. Identifying a novel mechanism, the dendritic retraction phenotype could be rescued by both overexpression of Gem, an inhibitor of RhoA, and treatment with $\mathrm{C} 3$ transferase, a Rho inhibitor.

\section{AS and Prader-Willi Syndrome}

Angelman syndrome (AS) and Prader-Willi syndrome (PWS) are neurodevelopmental disorders associated with genomic imprinting and ASD. AS and PWS are considered sister disorders, as they are both caused chromosomal deletion, or an imprinting defect, of the chromosomal region 15q11-13 [68, 69]. AS is a result of this deletion occurring on the maternal allele, causing the reduced expression of $U B E 3 A$ [68]. PWS is 
a caused by the deletion occurring on the paternal allele, resulting in the loss of or reduced expression of 7 paternally expressed genes in the affected $15 q$ region [70]. While AS and PWS do not have identical developmental defects, they do share neurologic symptoms such as cognitive, social, and speech disabilities [71, 72]. Chamberlain et al. [73] generated the first iPSC model of AS and PWS from patient cells. Their AS and PWS iPSCs showed no erasure of the DNA imprinting, and found that $U B E 3 A$ imprinting occurred during neuronal differentiation in the AS cells. [73]. However, the authors found no neuronal phenotypic differences between AS neurons and control neurons.

\section{PMDS}

PMDS, also known as $22 \mathrm{q} 13.3$ deletion syndrome, is a neurodevelopmental disorder characterized by a range of clinical symptoms, including absent or delayed speech, intellectual disability, mental retardation, and autism [74, 75]. PMDS is caused by deletion or loss of genes in the $22 \mathrm{q} 13$ region, typically causing loss of SHANK3. SH3 and multiple ankyrin repeat domains 3 is a scaffolding protein found in excitatory synapses involved in the organization of the postsynaptic density [76]. SHANK3 mutations have been associated with ASD, and mouse models carrying Shank3 mutations demonstrate synaptic defects and ASD-like behaviors [77, 78]. Shcheglovitov et al. [79] generated a PMDS iPSC model using fibroblasts from 2 patients with PMDS carrying large 22q13 deletions that include SHANK3 [79]. Neurons generated from the PMDS iPSCs demonstrated reduced excitatory synaptic transmission and fewer synapses. The authors revealed these neuronal defects could be rescued using expression of SHANK3 by lentivirus and by pharmacological treatment using insulin-like growth factor (IGF)-1 [79].

\section{RTT}

RTT is a monogenic progressive neurologic disorder caused by mutations in the X-linked gene MECP2 [80]. Patients with RTT are predominantly female, as affected males are usually preterm lethal and those that survive are severely affected [81, 82]. Patients with RTT have apparently normal development until they are 6-18 months old, which is followed by progressive neurologic abnormalities [83]. This period of regression is often characterized by the deceleration of head growth, and loss of acquired motor and language skills [83]. The spectrum of RTT neuropathology includes autistic behavior, stereotyped hand wringing, seizures, microcephaly, hypotonia, ataxia, and loss of speech $[84,85]$. Human postmortem analysis has revealed neuronal cellular phenotypes such as altered neuronal morphology, reduced soma size, fewer dendritic spines, and reduced dendritic arborization [86, 87]. Revealing the potential role of multiple cell types, several studies have demonstrated the effect of mutant astrocytes in RTT etiology [88-91]. In addition, recent reports have shown that microglia and oligodendrocytes are also important players in RTT pathophysiology [92-94].

While phenotypes have been robust and abundant, how alterations to $M E C P 2$ induce this array of abnormalities remains elusive. As a result, an abundant amount of research has been performed to study the function of $M E C P 2$. The causal role in RTT and the ability to rescue defects in RTT mouse models by reintroduction of Mecp 2 has demonstrated the importance of Mecp 2 to neuronal development and function [95, 96]. Methyl $\mathrm{CpG}$ binding protein 2 (MeCP2) has been shown to both activate and repress transcription [97]. Skene et al. [98] have also shown that MeCP2 is highly expressed in neurons and acts as a global transcriptional regulator, with a vast number of potential targets.

RTT has become a popular target, as several RTT iPSC studies have already been reported. Our work was the first to describe an iPSC model of RTT, where we discovered that neurons derived from RTT iPSC recapitulated several aspects of known RTT neuropathology [39]. The human neurons were derived from 4 different patients with RTT carrying different MECP2 mutations. The RTT neurons demonstrated phenotypes paralleling the human postmortem and rodent model findings, such as smaller soma size, reduced dendritic spine density, reduced spontaneous $\mathrm{Ca}^{2+}$ transient frequency, impaired excitatory synaptic transmission, and fewer excitatory synapses. To verify the causal role of $M E C P 2$, gain- and lossof-function assays using $M E C P 2$ re-expression and short hairpin RNA (shRNA) targeting $M E C P 2$ validated several of the neuronal abnormalities. Even more, treatment of the neurons with the candidate drug, IGF-1, was able to rescue the synaptic defects. IGF-1 is a known neurotrophic factor currently in clinical trials for RTT, and has been shown to be able to stimulate neuronal growth and synaptogenesis [99, 100]. Another report from our laboratory demonstrated that neural progenitor cells derived from RTT iPSCs had increased long interspersed element-1 retrotransposition, showing that MecP2 regulates these events [101]. A subsequent study using the RTT iPSC model also observed a reduced soma and nuclear size in affected neurons [102]. Kim et al. [103] observed a neuronal maturation defect in iPSCs derived from RTT. In a recent report, Williams et al. [104] generated astrocytes from RTT iPSCs and demonstrated that these mutant astrocytes and their conditioned media are enough to induce neuronal abnormalities. Using IGF-1 and GPE (an IGF-1 peptide), the authors were able, partially, to rescue the morphologic defects.

Exemplifying a prototypical iPSC model, RTT is a monogenetic disorder with known, robust phenotypes validated in numerous models. For RTT, iPSC modeling allowed for the ability to produce different affected subtypes that recapitulated known phenotypes, as well as for the generation of a drugscreening platform. 


\section{Modeling Nonsyndromic Autism}

A prevailing theme in the field is the use of syndromic forms of autism to shed light onto nonsyndromic autism. Because the genes driving syndromic autism are known, the idea is to determine how those known mutations induce neuronal phenotypes common among different ASDs, and subsequently examine if those same genes or pathways are affected in nonsyndromic cases of autism. Indeed, previous studies have shown shared synaptic phenotypes in syndromic and nonsyndromic mouse models of autism [105]. Because the large majority of ASD cases are sporadic, models of nonsyndromic autism are essential to the study of ASD etiology. ASDs exhibit a common core set of symptoms and demonstrate a strong genetic component, yet the exact etiology of ASD remains unknown [4]. ASD susceptibility has been implicated in several different chromosomal loci and genes, indicating genetic heterogeneity [106-108]. However, a large number of these genes are related and share molecular pathways, including those involved in neurotransmitter pathways [109-112], or neuron adhesion and junction molecules [113]. Mutations in $\mathrm{Ca}^{+2}$ channels and genes involved in $\mathrm{Ca}^{+2}$-regulated signaling have also been associated with ASD $[64,114,115]$. With increasing numbers of rare variants being implicated in ASD, but often presenting modest to low degrees of risk [10], it is crucial to identify and study the relevance of these rare variants to nonsyndromic ASD etiology.

As a proof-of-principle, our laboratory recently generated an iPSCs model of nonsyndromic autism to investigate cellular and molecular phenotypes [116]. The proband presented with classical autism, delayed motor skills development, and poor social responsiveness. In this model, we generated iPSCs from an ASD individual carrying a de novo balanced translocation disrupting TRPC6, which encodes for the protein channel transient receptor potential canonical 6 (TRPC6). This translocation resulted in TRPC6 haploinsufficiency the ASD individual (TRPC6-mut). Previously unassociated with ASD, TRPC6 is a voltage-independent, $\mathrm{Ca}^{2+}$-permeable cation channel. TRPC6 has been implicated in neuronal growth cone guidance, spinogenesis, and synaptogenesis, processes known to be affected in ASD [117-119]. Furthermore, TRPC6 has been shown to activate important pathways important for neuronal development and function, including the brain-derived neurotrophic factor (BDNF), calcium/calmodulin-dependent protein kinase IV, protein kinase $\mathrm{B}$, and cyclic adenosine monophosphate-response element binding protein phosphorylation signaling pathways (Fig. 1) [117, 120, 121]. Using iPSCs, we investigated the functional consequences of this TRPC6 haploinsufficiency.

Neurons derived from TRPC6-mut iPSCs revealed neuronal morphologic and functional alterations compared with control neurons. Global gene expression analysis of TRPC6-mut cells revealed that several cyclic adenosine monophosphateresponse element binding protein phosphorylation-targeted neuronal genes important for neurodevelopment were differentially regulated. Analysis of TRPC6-mut neurons demonstrated altered morphology, including reduced total length and dendritic arborization. Key neuronal functions were also affected, such as fewer dendritic spines and synapses, and impaired calcium dynamics. Importantly, these TRPC6-mut-dependent phenotypes were validated using shRNA targeting TRPC6, as well as re-expression of TRPC6 cDNA. Moreover, using shRNA targeting TRPC6 in mice, both in vivo and in vitro, demonstrated phenotypes paralleling the iPSC results, such as reduced neuronal arborization, and fewer spines and synapses. We were also able to rescue several of the neuronal abnormalities using the candidate drugs hyperforin and IGF-1. Our premise was that hyperforin, a specific activator of TRPC6 channels, might rescue phenotypes caused by haploinsufficiency by increasing TRPC6 signaling. As mentioned previously, IGF-1 has been used to rescue neuronal defects in RTT iPSCs models and is also used in ongoing clinical trials for ASD and other central nervous system disorders [39]. Hyperforin and IGF-1 were able to ameliorate neuronal complexity and increase dendritic spine density and synaptogenesis. Interestingly, we also observed that MecP2 affected TRPC6 expression and occupied the TRPC6 promoter region. This potential interaction reveals possible common pathways affected in syndromic and nonsyndromic ASD. Finally, to further investigate TRPC 6 as a novel ASDassociated gene, mutation analysis of sequencing data from 1041 individuals with ASD and 2872 controls revealed significantly more nonsynonymous mutations in the ASD population.

This study brings valuable information for ASD, as we demonstrated that an iPSC model of nonsyndromic ASD reveals striking neuronal phenotypes. These phenotypes and affected pathways represent potentially novel ASD biomarkers, and the ability to rescue these abnormalities provides the basis for potential drug screening platforms. While this study is the first to describe an iPSC model of nonsyndromic autism, numerous more lines from individuals with nonsyndromic ASD must be generated to validate common phenotypes and affected pathways, and to eventually create effective diagnostic tools.

\section{Common Mechanisms of Disease?}

The iPSC models of RTT, FXS, PMDS, and our nonsyndromic ASD model have demonstrated common phenotypes in neurons generated from these cell lines. All of these disorders demonstrated neuronal abnormalities such as altered morphology and synaptic deficits. And because all of these disorders fall under the umbrella of ASD, they share a common core set of symptoms. These observations suggest that there may be 


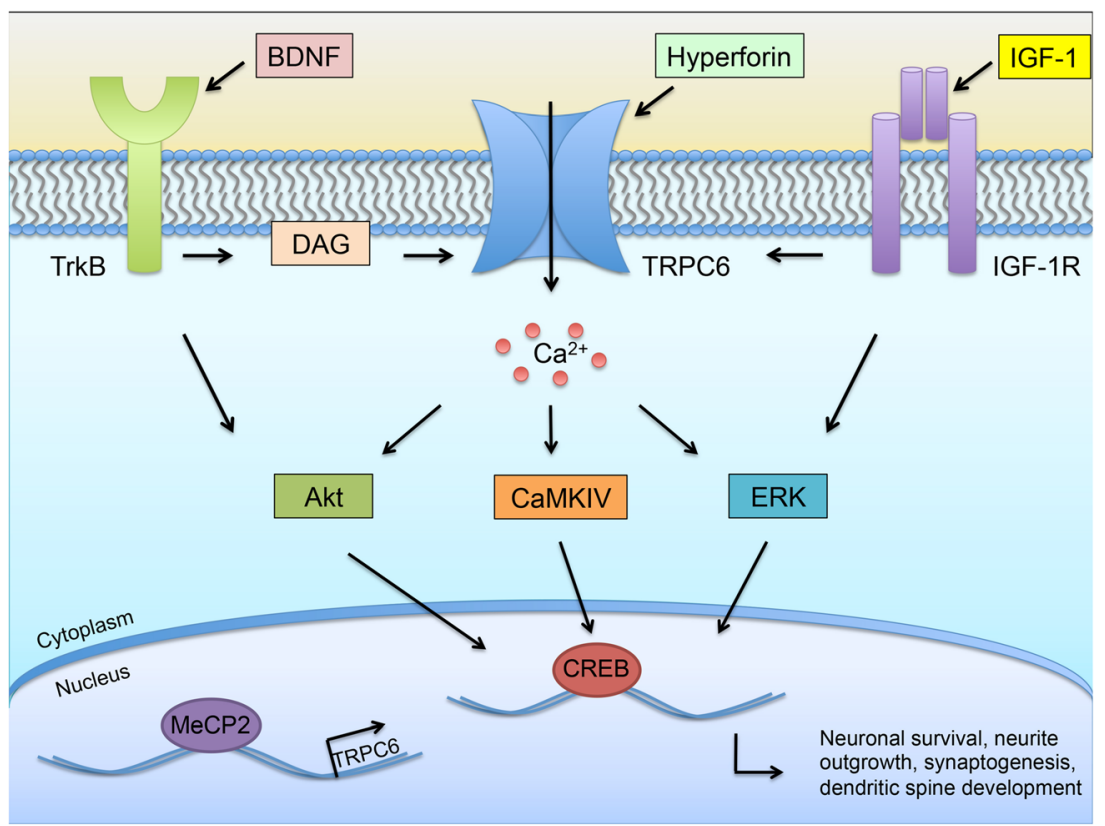

Fig. 1 Transient receptor potential canonical 6 (TRPC6) signaling pathways. TRPC6 has been implicated in the activation of several important neuronal signaling pathways. Influx of $\mathrm{Ca}^{2+}$ via TRPC6 pathways has been demonstrated to induce several signal transduction pathways, including the protein kinase B (Akt), calcium/calmodulindependent protein kinase IV (CaMKIV), and extracellular signalregulated kinase (ERK) pathways. Activation of these pathways eventually leads to the activation of the transcription factor cyclic adenosine monophosphate-response element binding protein phosphorylation (CREB), which has been demonstrated to promote proneuronal effects, including neuronal survival, neuronal plasticity, neurite outgrowth, and synaptogenesis. Several TRPC6 agonists have been demonstrated to stimulate TRPC6 and induce $\mathrm{Ca}^{2+}$ influx, including hyperforin and diacyl glycerol (DAG), the endogenous TRPC6 activator. Both the brain-derived neurotrophic factor (BDNF) receptor tropomyosin receptor kinase $\mathrm{B}(\mathrm{TrkB})$ and insulin-like growth factor (IGF)-1 receptor can promote downstream signaling that can also activate TRPC6, as well as both the ERK and Akt pathways. Recently, methyl $\mathrm{CpG}$ binding protein 2 (MeCP2) has been shown to bind to the promoter region of TRPC6 and to be important for TRPC6 expression common molecular mechanisms and pathways driving the observed phenotypes and symptoms in these disorders. Thus, iPSCs models represent a unique opportunity to examine and compare human neurons derived from different ASD at the cellular and molecular level.

Our recent report examined the potential relationship between MecP2 and TRPC6 [116]. MecP2 has previously been reported to affect TRPC6 levels [97]. Hippocampal neurons from RTT mouse models were reported to have impaired activity-dependent BNDF release and TRPC6 signaling [122]. Furthermore, TRPC6 has been shown to be necessary for certain BDNF-induced neurite growth cone guidance [117]. Indeed, MecP2 has been shown to regulate BDNF expression [123-125]. Another shared feature of these iPSCs models has been the ability of IGF-1 to rescue neuronal phenotypes, as IGF-1 treatment ameliorated the synaptic defects in RTT, PMDS, and nonsyndromic ASD iPSC-derived neurons. These findings suggest MecP2 and TRPC6 may be a part of common molecular pathway important for neuronal development and function (Fig. 1). The presence of common phenotypes, as well as shared mechanisms of drug rescue, implies common mechanisms and pathways driving pathogenesis among these different ASDs. More than anything, iPSC modeling provides the platform by which these analyses and comparisons can be made. However, more work needs to be done, as more models of ASD need to be generated in order to verify and better characterize genuine ASD cellular phenotypes.

\section{Limitations of iPSC Disease Modeling}

As with any model for disease, iPSC technology has definite limitations. To begin with, cells are grown in culture, which is a departure from true physiological conditions. As such, the components of the media may be overestimating or underestimating key signaling molecules, which can affect cell function. Thus, to verify that phenotypes observed in vitro are genuine, they must be validated in vivo or using other models. In addition, the field currently lacks the ability to generate a wide variety of specific neuronal subtypes. This is particularly important because certain neuronal subtypes are more severely affected by disorders such as ASD. For example, RTT pathophysiological studies revealed that the pyramidal neurons in cortical layer $\mathrm{V}$ are especially affected and display fewer dendritic spines [86]. Protocols are yet to be generated to produce such a specific subtype of cells. Another problem that arises because of this is the cellular 
heterogeneity in the iPSC-derived cultures. When generating cell types such as neurons, several other cell types remain present in the culture that could introduce artifacts. While some protocols exist for the fluorescence-associated cell sorting purification of cells such as neurons [126], more work is needed to be able to isolate all disease-relevant cell types. Another issue that arises with iPSC modeling is the problem of proper controls, especially with ASD. Currently, most reported ASD iPSC models use unaffected individuals or family members as controls. This can be problematic because each individual contains unique mutations and genetic differences that could potentially affect observable phenotypes. The ideal controlled experiment would be 2 lines containing the exact same genome, with the only difference being the mutation in question. Fortunately, the field is moving toward addressing this concern with the use of genome editing such as TALEN and CRISPR technology [127, 128]. Using genome editing, researchers are able to generate isogenic lines, differing only by the specific targeted mutation. This highlights another important consideration: the types of disorders iPSCs can effectively model. Certainly, iPSC modeling is most effective for genetic disorders with robust phenotypes. To maximize the potential of iPSCs, it is best if the genetics of the particular patient in question are known so that any phenotypes can be attributed to a specific genetic cause, and if phenotypes are robust enough or readily detectable

\section{Future Implications}

The iPSC-disease modeling strategy represents a significant step in ASD research and treatment. The most useful applications of these models are for the identification of cellular phenotypes, the elucidation of affected molecular pathways, and for the generation of new therapeutic strategies. This is particularly evident for syndromic forms of autism, as the genetics are known and can be attributed to the observed phenotypes. Yet our work denotes the first step in modeling nonsyndromic autism, which represents the majority of ASD but lacks clear, defining symptoms or cellular phenotypes. By taking advantage of next-generation genomics, one can map all of the genetic abnormalities and use iPSCs to analyze their impact on neuronal cells. Allowing for the development of personalized medicine, these iPSC models can take advantage of specific cellular phenotypes for drug screening purposes to identify potential therapeutic drugs tailored to an individual. While iPSC modeling shows great promise for ASD research, more work is needed. More iPSC models of nonsyndromic autism are necessary to generate a library of iPSC models from numerous autistic individuals to identify phenotypes and molecular pathways common to ASD. Fortunately, several outreach programs exist to facilitate community engagement and sample collection, such as the Tooth Fairy Project $[116,129]$. This project allows for families to send newly lost baby teeth from autistic individuals to researchers, from which dental pulp cells can be extracted and iPSCs generated. Finally, the comprehensive molecular and functional characterization of the iPSC-derived neurons from autistic individuals will be essential for the reliable discovery of true ASD phenotypes and molecular mechanisms driving ASD etiology.

Acknowledgments The work in the Muotri laboratory is supported by grants from the California Institute for Regenerative Medicine (CIRM) TR2-01814 and TR4-06747; the National Institutes of Health (NIH) through the NIH Director's New Innovator Award Program, 1-DP2OD006495-01 and R01MH094753; from the International Rett Syndrome Foundation (IRSF grant \# 2915); and NARSAD Independent Investigator.

Conflict of interest The authors declare no conflict of interest.

Required Author Forms Disclosure forms provided by the authors are available with the online version of this article.

\section{References}

1. Wing L, Gould J. Severe impairments of social interaction and associated abnormalities in children: epidemiology and classification. J Autism Dev Disord 1979;9:11-29.

2. American Psychiatric Association. Diagnostic and Statistical Manual of Mental Disorders, 5th Edition, American Psychiatric Association, Washington, DC.

3. State MW, Levitt P. The conundrums of understanding genetic risks for autism spectrum disorders. Nat Neurosci 2011;14:14991506.

4. Geschwind DH. Genetics of autism spectrum disorders. Trends Cogn Sci 2011;15:409-416.

5. Persico AM, Bourgeron T. Searching for ways out of the autism maze: genetic, epigenetic and environmental clues. Trends Neurosci 2006;29:349-358.

6. O'Roak BJ, Vives L, Fu W, et al. Multiplex targeted sequencing identifies recurrently mutated genes in autism spectrum disorders. Science 2012;338:1619-1622.

7. Jiang YH, Yuen RK, Jin X, et al. Detection of clinically relevant genetic variants in autism spectrum disorder by whole-genome sequencing. Am J Hum Genet 2013;93:249-263.

8. Iossifov I, O'Roak BJ, Sanders SJ, et al. The contribution of de novo coding mutations to autism spectrum disorder. Nature 2014;515:216-221.

9. De Rubeis S, He X, Goldberg AP, et al. Synaptic, transcriptional and chromatin genes disrupted in autism. Nature 2014;515:209215.

10. Geschwind DH. Autism: many genes, common pathways? Cell 2008;135:391-395.

11. Girirajan S, Rosenfeld JA, Coe BP, et al. Phenotypic heterogeneity of genomic disorders and rare copy-number variants. N Engl J Med 2012;367:1321-1331.

12. Steinberg J, Webber C. The roles of FMRP-regulated genes in autism spectrum disorder: single- and multiple-hit genetic etiologies. Am J Hum Genet 2013;93:825-839.

13. Leblond CS, Heinrich J, Delorme R, Proepper C, Betancur C, Huguet $\mathrm{G}$. Genetic and functional analyses of SHANK2 mutations suggest a multiple hit model of autism spectrum disorders. PLoS Genet 2012;8:e1002521. 
14. Ferner RE. Post-mortem clinical pharmacology. Br J Clin Pharmacol 2008;66:430-443.

15. Kim S, Webster MJ. Postmortem brain tissue for drug discovery in psychiatric research. Schizophr Bull 2009;35:1031-1033.

16. Nestler EJ, Hyman SE. Animal models of neuropsychiatric disorders. Nat Neurosci 2010;13:1161-1169.

17. Lewis MH, Tanimura Y, Lee LW, Bodfish JW. Animal models of restricted repetitive behavior in autism. Behav Brain Res 2007; 176:66-74.

18. Takahashi K, Yamanaka S. Induction of pluripotent stem cells from mouse embryonic and adult fibroblast cultures by defined factors. Cell 2006;126:663-676.

19. Soldner F, Jaenisch R. Medicine. iPSC disease modeling. Science 2012;338:1155-1156.

20. Park IH, Arora N, Huo H, et al. Disease-specific induced pluripotent stem cells. Cell 2008;134:877-886.

21. Chailangkarn T, Acab A, Muotri AR. Modeling neurodevelopmental disorders using human neurons. Curr Opin Neurobiol 2012;22:785-790.

22. Tiscornia G, Vivas EL, Izpisua Belmonte JC. Diseases in a dish: modeling human genetic disorders using induced pluripotent cells. Nat Med 2011;17:1570-1576.

23. Raya A, Rodriguez-Piza I, Guenechea G, et al. Disease-corrected haematopoietic progenitors from Fanconi anaemia induced pluripotent stem cells. Nature 2009;460:53-59.

24. Moretti A, Bellin M, Welling A, et al. Patient-specific induced pluripotent stem-cell models for long-QT syndrome. N Engl J Med 2010;363:1397-1409.

25. Itzhaki I, Maizels L, Huber I, et al. Modelling the long QT syndrome with induced pluripotent stem cells. Nature 2011;471:225229.

26. Carvajal-Vergara X, Sevilla A, D'Souza SL, et al. Patient-specific induced pluripotent stem-cell-derived models of LEOPARD syndrome. Nature 2010;465:808-812.

27. Marchetto MC, Brennand KJ, Boyer LF, Gage FH. Induced pluripotent stem cells (iPSCs) and neurological disease modeling: progress and promises. Hum Mol Genet 2011;20:R109-R115.

28. Dimos JT, Rodolfa KT, Niakan KK, et al. Induced pluripotent stem cells generated from patients with ALS can be differentiated into motor neurons. Science 2008;321:1218-1221.

29. Soldner F, Hockemeyer D, Beard C, et al. Parkinson's disease patient-derived induced pluripotent stem cells free of viral reprogramming factors. Cell 2009;136:964-977.

30. Israel MA, Yuan SH, Bardy C, et al. Probing sporadic and familial Alzheimer's disease using induced pluripotent stem cells. Nature 2012;482:216-220.

31. Mitne-Neto M, Machado-Costa M, Marchetto MC, et al. Downregulation of VAPB expression in motor neurons derived from induced pluripotent stem cells of ALS8 patients. Hum Mol Genet 2011;20:3642-3652.

32. Jiang H, Ren Y, Yuen EY, et al. Parkin controls dopamine utilization in human midbrain dopaminergic neurons derived from induced pluripotent stem cells. Nat Commun 2012;3:668.

33. Hardy JA, Higgins GA. Alzheimer's disease: the amyloid cascade hypothesis. Science 1992;256:184-185.

34. McLean CA, Cherny RA, Fraser FW, et al. Soluble pool of Abeta amyloid as a determinant of severity of neurodegeneration in Alzheimer's disease. Ann Neurol 1999;46:860-866.

35. Hardy J, Selkoe DJ. The amyloid hypothesis of Alzheimer's disease: progress and problems on the road to therapeutics. Science 2002;297:353-356.

36. Brennand KJ, Simone A, Jou J, et al. Modelling schizophrenia using human induced pluripotent stem cells. Nature 2011;473: 221-225.
37. Andrade LN, Nathanson JL, Yeo GW, Menck CF, Muotri AR. Evidence for premature aging due to oxidative stress in iPSCs from Cockayne syndrome. Hum Mol Genet 2012;21:3825-3834.

38. Urbach A, Bar-Nur O, Daley GQ, Benvenisty N. Differential modeling of fragile $\mathrm{X}$ syndrome by human embryonic stem cells and induced pluripotent stem cells. Cell Stem Cell 2010;6:407411.

39. Marchetto MC, Carromeu C, Acab A, Yu D, Yeo GW, Mu Y. A model for neural development and treatment of Rett syndrome using human induced pluripotent stem cells. Cell 2010;143:527539.

40. Goldstein S, Reynolds CR. Handbook of neurodevelopmental and genetic disorders in adults, Guilford Press, New York, 2005.

41. Mitchell KJ. The genetics of neurodevelopmental disease. Curr Opin Neurobiol 2011;21:197-203.

42. Zoghbi HY. Postnatal neurodevelopmental disorders: meeting at the synapse? Science 2003;302:826-830.

43. Weinberger DR. Implications of normal brain development for the pathogenesis of schizophrenia. Arch Gen Psychiatry 1987;44:660669.

44. Harrison PJ, Weinberger DR. Schizophrenia genes, gene expression, and neuropathology: on the matter of their convergence. Mol Psychiatry 2005;10:40-68.

45. Schizophrenia Psychiatric Genome-Wide Association Study C. Genome-wide association study identifies five new schizophrenia loci. Nat Genet 2011;43:969-976.

46. Ripke S, O'Dushlaine C, Chambert K, et al. Genome-wide association analysis identifies 13 new risk loci for schizophrenia. Nat Genet 2013;45:1150-1159.

47. Xu B, Roos JL, Dexheimer P, et al. Exome sequencing supports a de novo mutational paradigm for schizophrenia. Nat Genet 2011;43:864-868.

48. Murray RM, Lewis SW. Is schizophrenia a neurodevelopmental disorder? Br Med J (Clin Res Ed) 1987;295:681-682.

49. Thompson JL, Pogue-Geile MF, Grace AA. Developmental pathology, dopamine, and stress: a model for the age of onset of schizophrenia symptoms. Schizophr Bull 2004;30:875-900.

50. Rapoport JL, Giedd JN, Gogtay N. Neurodevelopmental model of schizophrenia: update 2012. Mol Psychiatry 2012;17:1228-1238.

51. Selemon LD, Goldman-Rakic PS. The reduced neuropil hypothesis: a circuit based model of schizophrenia. Biol Psychiatry 1999;45:17-25.

52. Jaaro-Peled H, Ayhan Y, Pletnikov MV, Sawa A. Review of pathological hallmarks of schizophrenia: comparison of genetic models with patients and nongenetic models. Schizophr Bull 2010;36:301-313.

53. Pasca SP, Portmann T, Voineagu I, et al. Using iPSC-derived neurons to uncover cellular phenotypes associated with Timothy syndrome. Nat Med 2011;17:1657-1662.

54. Amenduni M, De Filippis R, Cheung AY, et al. iPS cells to model CDKL5-related disorders. Eur J Hum Genet 2011;19:1246-1255.

55. Verkerk AJ, Pieretti M, Sutcliffe JS, et al. Identification of a gene (FMR-1) containing a CGG repeat coincident with a breakpoint cluster region exhibiting length variation in fragile $\mathrm{X}$ syndrome. Cell 1991;65:905-914.

56. Rogers SJ, Wehner DE, Hagerman R. The behavioral phenotype in fragile X: symptoms of autism in very young children with fragile X syndrome, idiopathic autism, and other developmental disorders. J Dev Behav Pediatr 2001;22:409-417.

57. Kindler S, Kreienkamp HJ. The role of the postsynaptic density in the pathology of the fragile X syndrome. Results Probl Cell Differ 2012;54:61-80.

58. Bassell GJ, Warren ST. Fragile X syndrome: loss of local mRNA regulation alters synaptic development and function. Neuron 2008;60:201-214. 
59. Eiges R, Urbach A, Malcov M, et al. Developmental study of fragile $\mathrm{X}$ syndrome using human embryonic stem cells derived from preimplantation genetically diagnosed embryos. Cell Stem Cell 2007;1:568-577.

60. Sheridan SD, Theriault KM, Reis SA, et al. Epigenetic characterization of the FMR1 gene and aberrant neurodevelopment in human induced pluripotent stem cell models of fragile $\mathrm{X}$ syndrome. PLoS One 2011;6:e26203.

61. Liu J, Koscielska KA, Cao Z, et al. Signaling defects in iPSCderived fragile X premutation neurons. Hum Mol Genet 2012;21: 3795-3805.

62. Hagerman R, Hagerman P. Advances in clinical and molecular understanding of the FMR1 premutation and fragile Xassociated tremor/ataxia syndrome. Lancet Neurol 2013;12:786798.

63. Doers ME, Musser MT, Nichol R, et al. iPSC-derived forebrain neurons from FXS individuals show defects in initial neurite outgrowth. Stem Cells Dev 2014;23:1777-1787.

64. Splawski I, Timothy KW, Sharpe LM, Decher N, Kumar P, Bloise R. $\mathrm{Ca}(\mathrm{V}) 1.2$ calcium channel dysfunction causes a multisystem disorder including arrhythmia and autism. Cell 2004;119:19-31.

65. Bader PL, Faizi M, Kim LH, et al. Mouse model of Timothy syndrome recapitulates triad of autistic traits. Proc Natl Acad Sci U S A 2011;108:15432-15437.

66. Barttfeld P, Wicker B, Cukier S, Navarta S, Lew S, Sigman M. A big-world network in ASD: dynamical connectivity analysis reflects a deficit in long-range connections and an excess of shortrange connections. Neuropsychologia 2011;49:254-263.

67. Krey JF, Pasca SP, Shcheglovitov A, et al. Timothy syndrome is associated with activity-dependent dendritic retraction in rodent and human neurons. Nat Neurosci 2013;16:201-209.

68. Knoll JH, Nicholls RD, Magenis RE, Graham JM, Jr., Lalande M, Latt SA. Angelman and Prader-Willi syndromes share a common chromosome 15 deletion but differ in parental origin of the deletion. Am J Med Genet 1989;32:285-290.

69. Kishino T, Lalande M, Wagstaff J. UBE3A/E6-AP mutations cause Angelman syndrome. Nat Genet 1997;15:70-73.

70. Bittel DC, Butler MG. Prader-Willi syndrome: clinical genetics, cytogenetics and molecular biology. Expert Rev Mol Med 2005;7: $1-20$.

71. Thibert RL, Larson AM, Hsieh DT, Raby AR, Thiele EA. Neurologic manifestations of Angelman syndrome. Pediatr Neurol 2013;48:271-279.

72. Whittington J, Holland A. Neurobehavioral phenotype in PraderWilli syndrome. Am J Med Genet C Semin Med Genet 2010;154C:438-447.

73. Chamberlain SJ, Chen PF, Ng KY, et al. Induced pluripotent stem cell models of the genomic imprinting disorders Angelman and Prader-Willi syndromes. Proc Natl Acad Sci U S A 2010;107: 17668-17673

74. Wilson HL, Wong AC, Shaw SR, et al. Molecular characterisation of the $22 \mathrm{q} 13$ deletion syndrome supports the role of haploinsufficiency of SHANK3/PROSAP2 in the major neurological symptoms. J Med Genet 2003;40:575-584.

75. Phelan K, McDermid HE. The 22q13.3 deletion syndrome (Phelan-McDermid syndrome). Mol Syndromol 2012;2:186-201.

76. Naisbitt S, Kim E, Tu JC, et al. Shank, a novel family of postsynaptic density proteins that binds to the NMDA receptor/PSD-95/ GKAP complex and cortactin. Neuron 1999;23:569-582.

77. Durand CM, Betancur C, Boeckers TM, Bockmann J, Chaste P, Fauchereau F. Mutations in the gene encoding the synaptic scaffolding protein SHANK3 are associated with autism spectrum disorders. Nat Genet 2007;39:25-27.

78. Wang X, McCoy PA, Rodriguiz RM, et al. Synaptic dysfunction and abnormal behaviors in mice lacking major isoforms of Shank3. Hum Mol Genet 2011;20:3093-3108.
79. Shcheglovitov A, Shcheglovitova O, Yazawa M, et al. SHANK3 and IGF1 restore synaptic deficits in neurons from 22q13 deletion syndrome patients. Nature 2013;503:267-271.

80. Amir RE, Van den Veyver IB, Wan M, Tran CQ, Francke U, Zoghbi HY. Rett syndrome is caused by mutations in X-linked MECP2, encoding methyl-CpG-binding protein 2. Nat Genet 1999;23:185-188.

81. Schanen C, Francke U. A severely affected male born into a Rett syndrome kindred supports X-linked inheritance and allows extension of the exclusion map. Am J Hum Genet 1998;63:267-269.

82. Villard L, Kpebe A, Cardoso C, Chelly PJ, Tardieu PM, Fontes M. Two affected boys in a Rett syndrome family: clinical and molecular findings. Neurology 2000;55:1188-1193.

83. Chahrour M, Zoghbi HY. The story of Rett syndrome: from clinic to neurobiology. Neuron 2007;56:422-437.

84. Williamson SL, Christodoulou J. Rett syndrome: new clinical and molecular insights. Eur J Hum Genet 2006;14:896-903.

85. Percy AK. Rett syndrome: exploring the autism link. Arch Neurol 2011;68:985-989.

86. Belichenko PV, Hagberg B, Dahlstrom A. Morphological study of neocortical areas in Rett syndrome. Acta Neuropathol 1997;93:5061.

87. Armstrong DD, Dunn K, Antalffy B. Decreased dendritic branching in frontal, motor and limbic cortex in Rett syndrome compared with trisomy 21. J Neuropathol Exp Neurol 1998;57: 1013-1017.

88. Ballas N, Lioy DT, Grunseich C, Mandel G. Non-cell autonomous influence of MeCP2-deficient glia on neuronal dendritic morphology. Nat Neurosci 2009;12:311-317.

89. Maezawa I, Swanberg S, Harvey D, LaSalle JM, Jin LW. Rett syndrome astrocytes are abnormal and spread MeCP2 deficiency through gap junctions. J Neurosci 2009;29:5051-5061.

90. Lioy DT, Garg SK, Monaghan CE, et al. A role for glia in the progression of Rett's syndrome. Nature 2011;475:497-500.

91. Okabe Y, Takahashi T, Mitsumasu C, Kosai K, Tanaka E, Matsuishi T. Alterations of gene expression and glutamate clearance in astrocytes derived from an MeCP2-null mouse model of Rett syndrome. PLoS One 2012;7:e35354.

92. Maezawa I, Jin LW. Rett syndrome microglia damage dendrites and synapses by the elevated release of glutamate. J Neurosci 2010;30:5346-5356.

93. Derecki NC, Cronk JC, Lu Z, et al. Wild-type microglia arrest pathology in a mouse model of Rett syndrome. Nature 2012;484:105-109.

94. Nguyen MV, Felice CA, Du F, et al. Oligodendrocyte lineage cells contribute unique features to Rett syndrome neuropathology. J Neurosci 2013;33:18764-18774.

95. Guy J, Hendrich B, Holmes M, Martin JE, Bird A. A mouse Mecp2-null mutation causes neurological symptoms that mimic Rett syndrome. Nat Genet 2001;27:322-326.

96. Guy J, Gan J, Selfridge J, Cobb S, Bird A. Reversal of neurological defects in a mouse model of Rett syndrome. Science 2007;315:1143-1147.

97. Chahrour M, Jung SY, Shaw C, Zhou X, Wong ST, Qin J. MeCP2, a key contributor to neurological disease, activates and represses transcription. Science 2008;320:1224-1229.

98. Skene PJ, Illingworth RS, Webb S, et al. Neuronal MeCP2 is expressed at near histone-octamer levels and globally alters the chromatin state. Mol Cell 2010;37:457-468.

99. Dudek H, Datta SR, Franke TF, et al. Regulation of neuronal survival by the serine-threonine protein kinase Akt. Science 1997;275:661-665.

100. O'Kusky JR, Ye P, D'Ercole AJ. Insulin-like growth factor-I promotes neurogenesis and synaptogenesis in the hippocampal dentate gyrus during postnatal development. J Neurosci 2000;20: 8435-8442. 
101. Muotri AR, Marchetto MC, Coufal NG, Oefner R, Yeo G, Nakashima K. L1 retrotransposition in neurons is modulated by MeCP2. Nature 2010;468:443-446.

102. Cheung AY, Horvath LM, Grafodatskaya D, et al. Isolation of MECP2-null Rett Syndrome patient hiPS cells and isogenic controls through X-chromosome inactivation. Hum Mol Genet 2011;20:2103-2115.

103. Kim KY, Hysolli E, Park IH. Neuronal maturation defect in induced pluripotent stem cells from patients with Rett syndrome. Proc Natl Acad Sci U S A 2011;108:14169-14174.

104. Williams EC, Zhong X, Mohamed A, et al. Mutant astrocytes differentiated from Rett syndrome patients-specific iPSCs have adverse effects on wild-type neurons. Hum Mol Genet 2014;23: 2968-2980.

105. Baudouin SJ. [Mouse models of autism: a common basis for syndromic and non syndromic autisms ?]. Med Sci (Paris) 2013;29:121-123 (in French).

106. Marshall CR, Noor A, Vincent JB, Lionel AC, Feuk L, Skaug J. Structural variation of chromosomes in autism spectrum disorder. Am J Hum Genet 2008;82:477-488.

107. Yonan AL, Alarcon M, Cheng R, et al. A genomewide screen of 345 families for autism-susceptibility loci. Am J Hum Genet 2003;73:886-897.

108. O'Roak BJ, Vives L, Girirajan S, Karakoc E, Krumm N, Coe BP. Sporadic autism exomes reveal a highly interconnected protein network of de novo mutations. Nature 2012;485:246-250.

109. Lawson-Yuen A, Saldivar JS, Sommer S, Picker J. Familial deletion within NLGN4 associated with autism and Tourette syndrome. Eur J Hum Genet 2008;16:614-618.

110. Rubenstein JL, Merzenich MM. Model of autism: increased ratio of excitation/inhibition in key neural systems. Genes Brain Behav 2003;2:255-267.

111. Jamain S, Betancur C, Quach H, et al. Linkage and association of the glutamate receptor 6 gene with autism. Mol Psychiatry 2002;7: 302-310.

112. Sudhof TC. Neuroligins and neurexins link synaptic function to cognitive disease. Nature 2008;455:903-911.

113. Rasin MR, Gazula VR, Breunig JJ, et al. Numb and Numbl are required for maintenance of cadherin-based adhesion and polarity of neural progenitors. Nat Neurosci 2007;10:819-827.

114. Hemara-Wahanui A, Berjukow S, Hope CI, Dearden PK, Wu SB, Wilson-Wheeler J. A CACNA1F mutation identified in an Xlinked retinal disorder shifts the voltage dependence of Cav1.4 channel activation. Proc Natl Acad Sci USA 2005;102:7553-7558.

115. Krey JF, Dolmetsch RE. Molecular mechanisms of autism: a possible role for $\mathrm{Ca} 2+$ signaling. Curr Opin Neurobiol 2007;17:112119.

116. Griesi-Oliveira K, Acab A, Gupta AR, et al. Modeling nonsyndromic autism and the impact of TRPC6 disruption in human neurons. Mol Psychiatry 2014 Nov 11.

117. Li Y, Jia YC, Cui K, Li N, Zheng ZY, Wang YZ. Essential role of TRPC channels in the guidance of nerve growth cones by brainderived neurotrophic factor. Nature 2005;434:894-898.

118. Zhou J, Du W, Zhou K, Tai Y, Yao H, Jia Y. Critical role of TRPC6 channels in the formation of excitatory synapses. Nat Neurosci 2008; 11:741-743.
119. Leuner K, Li W, Amaral MD, Rudolph S, Calfa G, Schuwald AM. Hyperforin modulates dendritic spine morphology in hippocampal pyramidal neurons by activating $\mathrm{Ca}(2+)$-permeable TRPC6 channels. Hippocampus 2012;23:40-52.

120. Tai Y, Feng S, Ge R, Du W, Zhang X, He Z. TRPC6 channels promote dendritic growth via the CaMKIV-CREB pathway. J Cell Sci 2008;121:2301-2307.

121. Heiser JH, Schuwald AM, Sillani G, Ye L, Muller WE, Leuner K. TRPC6 channel-mediated neurite outgrowth in PC12 cells and hippocampal neurons involves activation of RAS/MEK/ERK, PI3K, and CAMKIV signaling. J Neurochem 2013;127:303-313.

122. Li W, Calfa G, Larimore J, Pozzo-Miller L. Activity-dependent BDNF release and TRPC signaling is impaired in hippocampal neurons of Mecp2 mutant mice. Proc Natl Acad Sci USA 2012;109:17087-17092.

123. Im HI, Hollander JA, Bali P, Kenny PJ. MeCP2 controls BDNF expression and cocaine intake through homeostatic interactions with microRNA-212. Nat Neurosci 2010;13:1120-1127.

124. Kline DD, Ogier M, Kunze DL, Katz DM. Exogenous brainderived neurotrophic factor rescues synaptic dysfunction in Mecp2-null mice. J Neurosci 2010;30:5303-5310.

125. Zhou Z, Hong EJ, Cohen S, et al. Brain-specific phosphorylation of $\mathrm{MeCP} 2$ regulates activity-dependent Bdnf transcription, dendritic growth, and spine maturation. Neuron 2006;52:255-269.

126. Yuan SH, Martin J, Elia J, et al. Cell-surface marker signatures for the isolation of neural stem cells, glia and neurons derived from human pluripotent stem cells. PLoS One 2011;6:e17540.

127. Miller JC, Tan S, Qiao G, et al. A TALE nuclease architecture for efficient genome editing. Nat Biotechnol 2011;29:143-148.

128. Mali P, Yang L, Esvelt KM, et al. RNA-guided human genome engineering via Cas9. Science 2013;339:823-826.

129. Beltrao-Braga PI, Pignatari GC, Maiorka PC, Oliveira NA, Lizier NF, Wenceslau CV. Feeder-free derivation of induced pluripotent stem cells from human immature dental pulp stem cells. Cell Transplant 2011;20:1707-1719.

130. Li LB, Chang KH, Wang PR, Hirata RK, Papayannopoulou T, Russell DW. Trisomy correction in Down syndrome induced pluripotent stem cells. Cell Stem Cell 2012;11:615-619.

131. Shi Y, Kirwan P, Smith J, MacLean G, Orkin SH, Livesey FJ. A human stem cell model of early Alzheimer's disease pathology in Down syndrome. Sci Transl Med 2012;4:124ra29.

132. Lu HE, Yang YC, Chen SM, et al. Modeling neurogenesis impairment in Down syndrome with induced pluripotent stem cells from Trisomy 21 amniotic fluid cells. Exp Cell Res 2013;319:498-505.

133. Weick JP, Held DL, Bonadurer GF, 3rd, et al. Deficits in human trisomy 21 iPSCs and neurons. Proc Natl Acad Sci U S A 2013;110:9962-9967.

134. Hibaoui Y, Grad I, Letourneau A, et al. Modelling and rescuing neurodevelopmental defect of Down syndrome using induced pluripotent stem cells from monozygotic twins discordant for trisomy 21. EMBO Mol Med 2014;6:259-277.

135. Chen C, Jiang P, Xue H, et al. Role of astroglia in Down's syndrome revealed by patient-derived human-induced pluripotent stem cells. Nat Commun 2014;5:4430.

136. Yang J, Cai J, Zhang Y, et al. Induced pluripotent stem cells can be used to model the genomic imprinting disorder Prader-Willi syndrome. J Biol Chem 2010;285:40303-11. 